\title{
Supporting Information: Dynamics of Flexible Viruses in Polymer Solutions
}

Maxwell Smith, ${ }^{\dagger}$ Ryan Poling-Skutvik,,$^{\ddagger}$ Ali H. Slim, ${ }^{\dagger}$ Richard C. Willson, ${ }^{*, \dagger}$ and Jacinta C. Conrad*,†

$\dagger$ Department of Chemical and Biomolecular Engineering, University of Houston, Houston, $T X 77204-4004$

$\ddagger$ Department of Chemical Engineering, University of Rhode Island, Kingston, RI 02881

E-mail: willson@uh.edu; jcconrad@uh.edu

\section{Polymer Rheology}

We prepared a series of dilute solutions of partially hydrolyzed polyacrylamide (HPAM, $M_{\mathrm{w}}=8 \times 10^{6} \mathrm{~g} / \mathrm{mol}$ ) in $1 \mathrm{x}$ PBS from a concentrated stock solution and homogenized the samples using a roller mixer overnight at room temperature. The viscosities of these solutions were measured at room temperature using an Ubbelohde viscometer (0C, Cannon Instruments Company). We extracted the intrinsic viscosity $[\eta]=3.2 \pm 0.1 \mathrm{~L} / \mathrm{g}$ from $\eta=\eta_{0}\left(1+[\eta] c_{\mathrm{HPAM}}\right)$, where $\eta_{0}$ is the solvent viscosity. We then calculated the overlap concentration $c^{*}=1 /[\eta]=0.31 \pm 0.01 \mathrm{~g} / \mathrm{L}$ and the radius of gyration $R_{\mathrm{g}, 0}=$ $\left(M_{\mathrm{W}} /\left(4 / 3 \pi N_{\mathrm{A}} c^{*}\right)\right)^{1 / 3}=220 \pm 20 \mathrm{~nm}$, where $N_{\mathrm{A}}$ is Avogadro's number. We calculated the polymer correlation length $\xi$ as a function of polymer concentration normalized by overlap concentration $c / c^{*}, \xi=R_{\mathrm{g}, 0}\left(c / c^{*}\right)^{(-\nu /(3 \nu-1))}$, where $\nu=0.588$ is the Flory exponent in good solvent conditions.

We characterized the viscosity of more concentrated solutions of HPAM in 1x PBS using a 


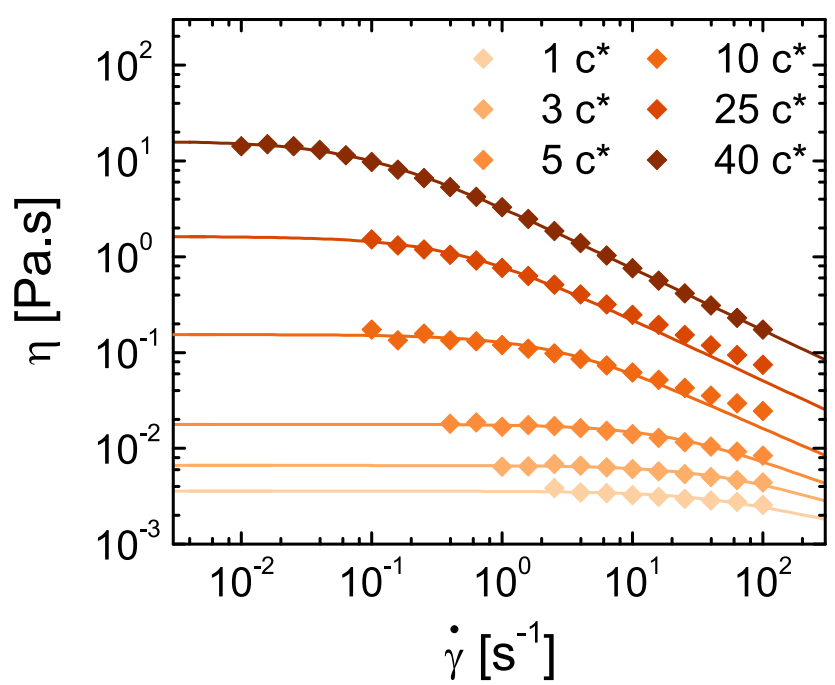

Figure S1: Viscosity $\eta$ as a function of shear rate $\dot{\gamma}$ at various HPAM concentrations $c$. Solid curves represent Cross-Carreau-Yasuda model fits of $\eta$ at each $c$.

Discovery Hybrid Rheometer (DHR-2, TA Instruments) using a concentric cylinder geometry with a bob length of $42 \mathrm{~mm}$ at $20{ }^{\circ} \mathrm{C}$. The viscosities of the HPAM solutions decreased with increasing shear rate $\dot{\gamma}$ and were well represented by the Cross-Carreau-Yasuda model, ${ }^{1}$

$$
\eta=\left(\eta_{\mathrm{s}}-\eta_{\infty}\right)(1+\tau \dot{\gamma})^{n-1}+\eta_{\infty}
$$

where $\eta_{\mathrm{s}}$ is the zero-shear viscosity, $\eta_{\infty}$ is the infinite-shear viscosity, $\tau$ is the relaxation time of the polymer, and $n$ is the power-law decay exponent. From these fits, we extracted $\eta_{0}$ and calculated the specific viscosity $\eta_{\mathrm{SP}}=\eta_{\mathrm{s}}-\eta_{0}$ as a function of $c$. We combined the data from the intrinsic viscosity measurements and the rheometer measurements into a single plot (Figure S2). We then compared the data to the predicted scaling behavior of $\eta_{\mathrm{SP}}$ in different polymer concentration regimes. In the dilute limit, $c<c^{*}$, the scaling exponent was 1 . In the semidilute limit, $c^{*}<c<c_{\mathrm{E}}$, the scaling exponent was 2 . In the entangled limit, $c>c_{\mathrm{E}}$, the scaling exponent was $14 / 3 .^{1}$ The measurements followed the predicted scaling well in all the concentration regimes, and from this data, we estimated the entanglement concentration to be approximately $20 c^{*}$. 


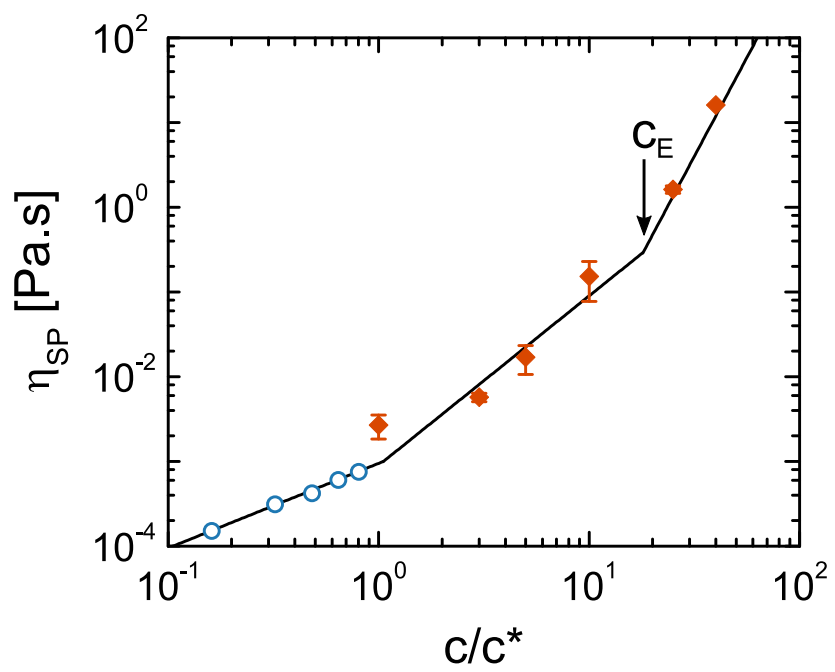

Figure S2: Specific viscosity $\eta_{\mathrm{SP}}$ measured by Ubbelohde viscometer (blue open) and Discovery Hybrid Rheometer (orange closed) as a function of of polymer concentration $c / c^{*}$. Solid lines represent predicted scaling behavior of $\eta_{\mathrm{SP}}$ at varying $c / c^{*}$ with arrow indicating crossover from semidilute to entangled regime.

\section{Polymer Time Scales}

At length scales smaller than the polymer correlation length $\xi$, polymer relaxations are described by the Zimm model with a characteristic relaxation time $\tau_{\xi} \sim \eta_{\mathrm{s}} \xi^{3} / k_{\mathrm{B}} T$. At larger time scales $t>\tau_{\xi}$, the polymer chain relaxes as a chain of correlation blobs until the terminal relaxation time $\tau_{\mathrm{R}} \sim \tau_{\xi}(N / g)^{2 \nu+1}$, where $N / g$ is the number of correlation blobs per polymer chain, and $\nu=0.588$ is the Flory exponent in good solvent conditions. Estimates of these time scales are given in Table S1.

Table S1: Zimm $\tau_{\xi}$ and Rouse $\tau_{\mathrm{R}}$ polymer relaxation times as a function of normalized polymer concentration $c / c^{*}$.

\begin{tabular}{|c|c|c|}
\hline Polymer concentration $c / c^{*}$ & $\tau_{\xi}[\mathrm{s}]$ & $\tau_{\mathrm{R}}[\mathrm{s}]$ \\
\hline 1.0 & $25 . \times 10^{-5}$ & 0.009 \\
\hline 2.2 & $4.1 \times 10^{-5}$ & 0.017 \\
\hline 5.2 & $0.56 \times 10^{-5}$ & 0.032 \\
\hline 9.8 & $0.13 \times 10^{-5}$ & 0.053 \\
\hline
\end{tabular}




\section{Virus Relaxation Times}

We estimated the virus particle diffusion time $\tau_{\mathrm{D}}=S_{\mathrm{i}}^{2} / D_{\mathrm{t}}$, where $S_{\mathrm{i}}$ is a virus length scale, and $D_{\mathrm{t}}$ is the virus diffusivity at polymer concentration $c$. Estimates of $\tau_{\mathrm{D}}$ for various characteristic virus length scales, based on the length $L$ and radius $R$, are provided for PVM (Table S2), M13 (Table S3), and pf1 (Table S4).

Table S2: Diffusive time scales $\tau_{\mathrm{D}}$ for different PVM length scales $S_{\mathrm{i}}$ as a function of normalized polymer concentration $c / c^{*}$.

\begin{tabular}{|c|c|c|c|c|c|c|}
\hline$c / c^{*}$ & \multicolumn{6}{|c|}{$\operatorname{PVM}(200 \mathrm{~nm}, 13 \mathrm{~nm}) \tau_{\mathrm{D}}[\mathrm{s}]$} \\
\hline & $L$ & $R$ & $L \ln (L / R)^{-1}$ & $R \ln (L / R)^{-1}$ & $R_{\mathrm{V}}$ & $R_{\mathrm{SA}}$ \\
\hline 1.0 & 0.032 & $1.4 \times 10^{-4}$ & 0.0028 & $1.2 \times 10^{-5}$ & 0.0007 & 0.0010 \\
\hline 2.2 & 0.055 & $2.3 \times 10^{-4}$ & 0.0048 & $2.0 \times 10^{-5}$ & 0.0012 & 0.0018 \\
\hline 5.2 & 0.13 & $5.3 \times 10^{-4}$ & 0.011 & $4.7 \times 10^{-5}$ & 0.0027 & 0.0041 \\
\hline 9.8 & 0.27 & $11 . \times 10^{-4}$ & 0.023 & $9.9 \times 10^{-5}$ & 0.0058 & 0.0087 \\
\hline
\end{tabular}

Table S3: Diffusive time scales $\tau_{\mathrm{D}}$ for different M13 length scales $S_{\mathrm{i}}$ as a function of normalized polymer concentration $c / c^{*}$.

\begin{tabular}{|c|c|c|c|c|c|c|}
\hline$c / c^{*}$ & \multicolumn{6}{|c|}{$\mathrm{M} 13(900 \mathrm{~nm}, 6 \mathrm{~nm}) \tau_{\mathrm{D}}[\mathrm{s}]$} \\
\hline & $L$ & $R$ & $L \ln (L / R)^{-1}$ & $R \ln (L / R)^{-1}$ & $R_{\mathrm{V}}$ & $R_{\mathrm{SA}}$ \\
\hline 1.3 & 1.3 & $0.56 \times 10^{-4}$ & 0.039 & $0.17 \times 10^{-5}$ & 0.0013 & 0.0042 \\
\hline 2.2 & 1.4 & $0.63 \times 10^{-4}$ & 0.044 & $0.19 \times 10^{-5}$ & 0.0015 & 0.0047 \\
\hline 5.7 & 4.3 & $1.9 \times 10^{-4}$ & 0.13 & $0.58 \times 10^{-5}$ & 0.0044 & 0.014 \\
\hline 10. & 8.0 & $3.6 \times 10^{-4}$ & 0.25 & $1.1 \times 10^{-5}$ & 0.0083 & 0.027 \\
\hline
\end{tabular}

Table S4: Diffusive time scales $\tau_{\mathrm{D}}$ for different pf1 length scales $S_{\mathrm{i}}$ as a function of normalized polymer concentration $c / c^{*}$.

\begin{tabular}{|c|c|c|c|c|c|c|}
\hline$c / c^{*}$ & \multicolumn{6}{|c|}{$\operatorname{pf}(2000 \mathrm{~nm}, 6 \mathrm{~nm}) \tau_{\mathrm{D}}[\mathrm{s}]$} \\
\hline & $L$ & $R$ & $L \ln (L / R)^{-1}$ & $R \ln (L / R)^{-1}$ & $R_{\mathrm{V}}$ & $R_{\mathrm{SA}}$ \\
\hline 1.1 & 6.4 & $0.58 \times 10^{-4}$ & 0.16 & $0.14 \times 10^{-5}$ & 0.0023 & 0.0096 \\
\hline 2.1 & 8.5 & $0.77 \times 10^{-4}$ & 0.21 & $0.18 \times 10^{-5}$ & 0.0030 & 0.013 \\
\hline 4.8 & 15. & $1.4 \times 10^{-4}$ & 0.37 & $0.33 \times 10^{-5}$ & 0.0055 & 0.023 \\
\hline
\end{tabular}




\section{Spherical Particle Tracking}

Using fluorescence microscopy and particle tracking algorithms as described in the main text, we measured the dynamics of spherical red fluorescent polystyrene nanoparticles (PS) of diameter $100 \mathrm{~nm}, 300 \mathrm{~nm}$, and $2 \mu \mathrm{m}$ (ThermoFisher Scientific) as a function of HPAM concentration $c$. Mean-squared displacements (MSDs) $\left\langle\Delta x^{2}\right\rangle$ scaled linearly for all particle sizes and polymer concentrations, indicating diffusive motion, but decreased with increasing $c$ as the solution viscosity increased (Figure S3).

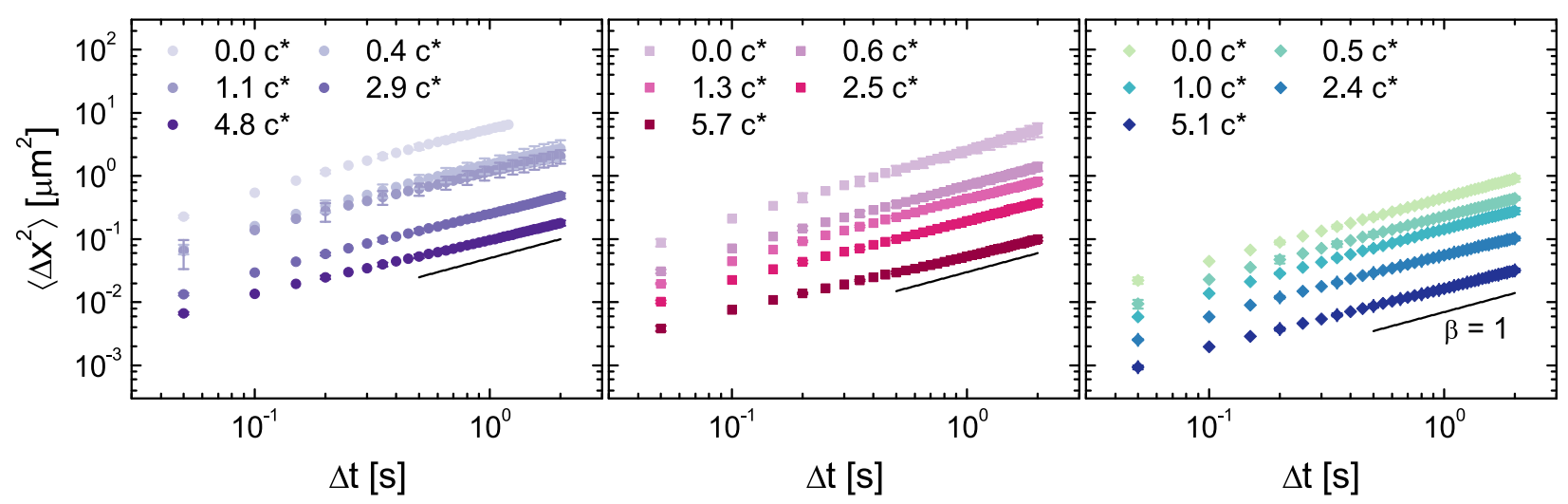

Figure S3: Mean-squared displacements $\left\langle\Delta x^{2}\right\rangle$ as a function of lag time $\Delta t$ for spherical particles (a) $100 \mathrm{~nm}$, (b) $300 \mathrm{~nm}$, and (c) $2 \mu \mathrm{m}$ at various polymer concentrations $c$ as a function of lag time $\Delta t$. Solid reference lines represent linear scaling.

The probability distributions of displacements (PDDs) $G_{\mathrm{s}}$ as a function of normalized displacement $\Delta x\left(2 D_{\mathrm{t}} \Delta t\right)^{-1 / 2}$ followed a Gaussian distribution for all sizes of spherical particles and all $c$. All PDDs for each PS also collapsed onto the same distribution, indicating similar Gaussian dynamics for each PS as $c$ varied (Figure S4). 


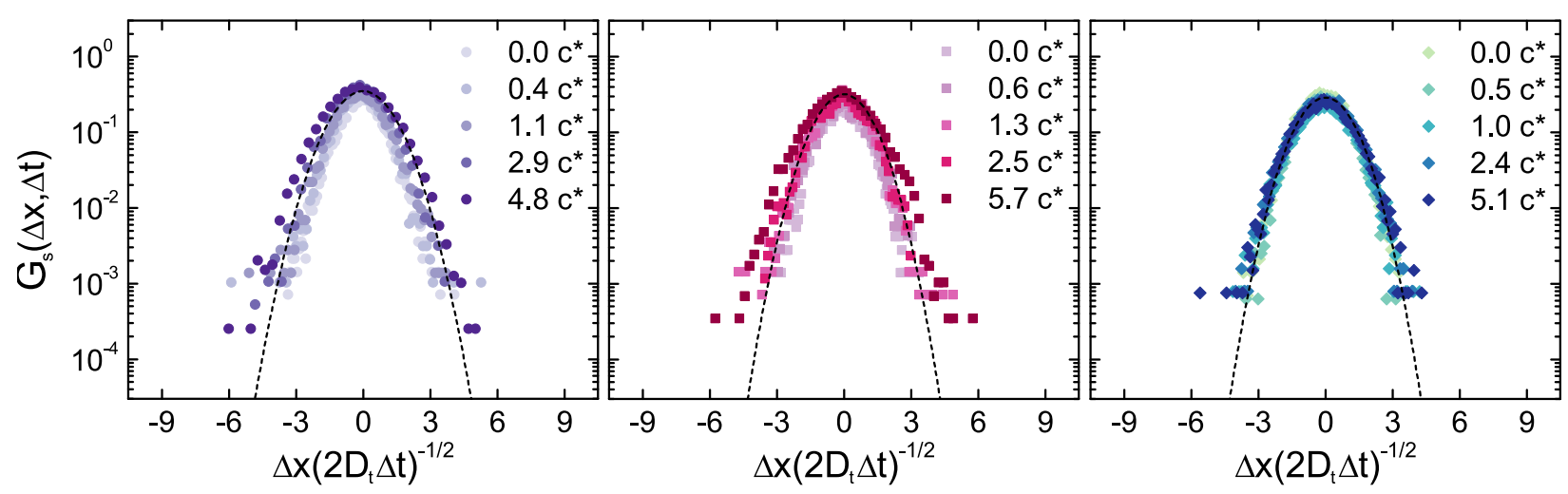

Figure S4: Normalized probability distributions of displacements $G_{\mathrm{s}}(\Delta x, \Delta t)$ for spherical particles (a) $100 \mathrm{~nm}$, (b) $300 \mathrm{~nm}$, and (c) $2 \mu \mathrm{m}$ at $\Delta t=0.05 \mathrm{~s}$ for varying polymer concentrations $c$ as a function of the normalized displacement $\Delta x\left(2 D_{\mathrm{t}} \Delta t\right)^{-1 / 2}$. The dashed lines represent Gaussian distributions. 


\section{Dynamic Light Scattering}

Dynamic light scattering (DLS) was used to measure the change in hydrodynamic radius $R_{\mathrm{H}}$ of PS in the presence of HPAM in 1x PBS. The light-scattering setup consisted of an ALV goniometer, a helium-neon laser at wavelength $632.8 \mathrm{~nm}$, and an ALV-5000/EPP Multiple tau Digital Correlator (ALV-GmbH, Langen, Germany). Four samples were tested with 100 $\mathrm{nm}$ and $300 \mathrm{~nm}$ PS in 1x PBS and 0.1 $c^{*}$ HPAM in 1x PBS and were placed in cleaned, 10 $\mathrm{mm}$ cylindrical cuvettes. Scattering data was collected from five scattering angles: $120^{\circ}$, $105^{\circ}, 90^{\circ}, 75^{\circ}$, and $60^{\circ}$, for $15 \mathrm{~s}$ at $20^{\circ} \mathrm{C}$. We fitted the intensity-intensity autocorrelation function data $G_{2}-1$ as a function of lag time $\Delta t$ using the method of cumulants up to the third moment, ${ }^{2}$

$$
G_{2}(\Delta t, q)-1=A+B \exp (-2 \Gamma(q) \Delta t)\left(1+\frac{\mu_{2}}{2}-\frac{\mu_{3}}{6}\right)^{2}
$$

where $\Gamma$ is the average relaxation time, and $\mu_{i}$ is the $i$ th moment. We then plotted $\Gamma$ as a function of the wave vector squared $Q^{2}=(4 \pi n \sin (\theta / 2) / \lambda)^{2}$, where $n$ is the refractive index of the solution, $\theta$ is the scattering angle, and $\lambda$ is the wavelength of the scattering source (Figure S5). The linear fit of this plot corresponded to the translational diffusivity $D_{\mathrm{t}}=\Gamma / Q^{2}$. Through the Stokes-Einstein relation, $D_{\mathrm{t}}$ is inversely proportional to the product of $\eta$ and $R_{\mathrm{H}}, D_{\mathrm{t}} \propto 1 / \eta R_{\mathrm{H}}$. With polymer, $\eta$ of the samples slightly increased which decreased $D_{\mathrm{t}}$. By accounting for this change, $R_{\mathrm{H}}$ of PS was extracted from the linear slope and was similar with and without HPAM. We concluded, therefore, that the polymer chains did not significantly adsorb on PS spheres. 


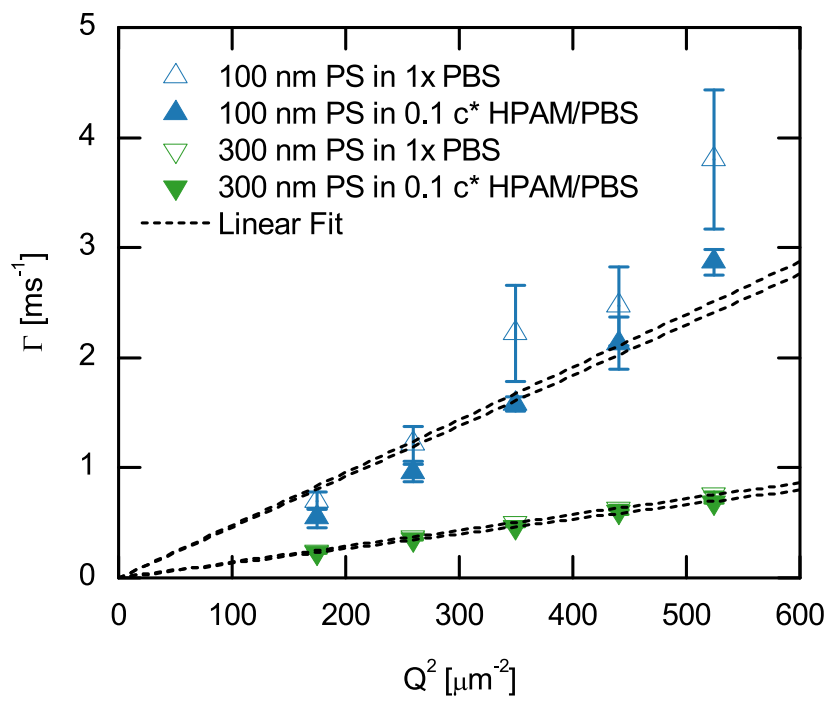

Figure S5: Relaxation time $\bar{\Gamma}$ of $100 \mathrm{~nm}$ PS and $300 \mathrm{~nm}$ PS in $1 \mathrm{x}$ PBS and $0.1 c^{*} \mathrm{HPAM}$ in $1 \mathrm{x}$ PBS as a function of wave vector squared $Q^{2}$. Dashed line represents a linear fit.

\section{References}

(1) Bird, R. B.; Armstrong, R. C.; Hassager, O. Dynamics of Polymeric Liquids: Volume 1, 2nd ed.; John Wiley \& Sons: New York, 1987; p 672.

(2) Frisken, B. J. Revisiting the method of cumulants for the analysis of dynamic lightscattering data. Applied Optics 2001, 40, 4087-4091. 